\title{
Reflets
}

Revue d'intervention sociale et communautaire

\section{Paternités méconnues}

\section{Jean-Martin Deslauriers}

Volume 16, numéro 1, printemps 2010

Paternités méconnues (Deuxième partie)

URI : https://id.erudit.org/iderudit/044439ar

DOI : https://doi.org/10.7202/044439ar

Aller au sommaire du numéro

Éditeur(s)

Reflets : Revue d'intervention sociale et communautaire

ISSN

1203-4576 (imprimé)

1712-8498 (numérique)

Découvrir la revue

Citer ce document

Deslauriers, J.-M. (2010). Paternités méconnues. Reflets, 16(1), 10-16.

https://doi.org/10.7202/044439ar

Tous droits réservés @ Reflets : Revue d'intervention sociale et communautaire, 2010

dectén services d'Érudit (y compris la reproduction) est assujettie à sa politique d'utilisation que vous pouvez consulter en ligne.

https://apropos.erudit.org/fr/usagers/politique-dutilisation/ 


\section{Paternités méconnues}

Jean-Martin Deslauriers

Professeur, École de service social, Université d'Ottawa

Ce numéro constitue la deuxième partie du dossier de la revue Reflets sur les perspectives et les enjeux liés à la paternité. Après avoir situé dans le premier numéro la paternité comme objet de recherche, de réflexion théorique et pratique, nous en abordons maintenant certains contextes et enjeux sous des perspectives peu étudiées à ce jour. Le champ de recherche sur la paternité connait une diversité proportionnelle aux conditions de son exercice, certaines d'entre elles n'étant vécues parfois que par une faible proportion de la population. D'où la nécessité de présenter la paternité sous ses formes diverses. La recherche a permis jusqu'ici de documenter le sujet sous plusieurs aspects théoriques, et ce, à partir de l'expérience de pères ainsi que de leurs rapports avec les services sociaux et de santé. Cependant, comme pour plusieurs phénomènes sociaux, les manifestations les moins fréquentes de la paternité sont peu étudiées et constituent en elles-mêmes des pans entiers de recherches potentielles.

En soulevant de nouveaux angles d'approche de la paternité ou des situations particulières vécues par des pères, ce numéro fait donc place " aux paternités » dans leur diversité, et tout particulièrement, à ces paternités qualifiées de « méconnues » du fait qu'elles s'appliquent à des vécus paternels moins courants dans notre société et à d'autres qui s'éloignent parfois des valeurs et des normes sociales en vigueur.

La production d'un numéro de revue impose malheureusement des limites à la quantité d'articles à y être publiés. En effet, plusieurs autres aspects méconnus de la paternité auraient pu être mis en lumière, tels que la paternité en contexte de handicap ou de déficience (celui du père ou de l'enfant), les pères monoparentaux, 
les pères d'adoption de même que les pères homosexuels ou encore, les situations de paternité tardive. Mais en présentant dans ce numéro certaines situations vécues par des pères atypiques, nous espérons contribuer à formuler une approche familiale de leur situation et des possibilités de politiques sociales.

Parmi les perspectives d'étude des paternités souvent négligées dans la recherche, on retrouve les contextes dans lesquels des enfants sont témoins ou subissent différents types d'abus.Ajoutons d'autres exemples de ces angles morts de la paternité :les contextes de désengagement paternel, de violence conjugale et familiale, de toxicomanie, de jeu pathologique et d'incarcération. Dans ces conditions, le père, considéré comme " toxique " pour ses enfants, suscite moins d'intérêt comme enjeu social, comme objet de recherche et de réflexion sur les plans des pratiques sociales. Pourtant, il est pertinent de se pencher sur différentes réalités liées à l'exercice de ce rôle pour, graduellement, reconnaitre des dimensions moins fréquentes, qui suscitent moins de sympathie, qui sont moins documentées et souvent méconnues des services sociaux. Selon nous, il incombe à la communauté des chercheurs de documenter ces contextes et de réfléchir aux solutions possibles. Certains pères ne reçoivent que peu de soutien quand ils vivent des difficultés. Ce n'est que par le truchement de différentes lois et dispositions légales que l'État intervient et, le plus souvent, lorsque ces pères ont posé des gestes répréhensibles. Plus rarement en amont.

Pourtant, ces situations particulières méritent que l'on s'y attarde non seulement pour soutenir les pères, mais aussi les mères et les enfants qui les partagent. S'il y a des paternités méconnues, il y a des enfances oubliées. S'interroger sur des nouvelles pratiques sociales pour mieux intervenir auprès des pères, c'est potentiellement aider des enfants qui présentent des besoins particuliers et nécessitent une intervention adaptée à leur réalité.

Avant d'aborder certaines situations particulières d'exercice de la paternité, un regard plus large sur la question est proposé. À l'ouverture, le numéro présente une entrevue réalisée par Madeleine Dubois auprès de Tim Paquette, coordonnateur 
sortant du Réseau ontarien Initiative pour l'engagement paternel, un programme provincial de promotion de la paternité. Cet entretien a la grande qualité de présenter sur la paternité une réflexion qui allie théorie et pratique. On y développe une discussion qui tient compte de la place des pères dans notre société, des rapports hommes/femmes de la socialisation masculine tout en liant ces enjeux aux pratiques auprès des pères au sein des services sociaux. Également, cet invité de Reflets souligne le décalage entre les nouveaux phénomènes sociaux concernant la paternité et la réponse des services sociaux à ces derniers. Parmi eux, des contextes méconnus de la paternité tels que la monoparentalité au masculin. Enfin, l'interviewé avance des propositions de pratiques sociales touchant les dimensions individuelles, familiales et sociales de la paternité.

L'article de Josée St-Denis et de Nérée St-Amand porte sur des enjeux sociaux reliés à la paternité, lesquels sont situés dans une perspective très large. Les auteurs rappellent d'abord quelques volets de l'histoire de la paternité au cours de la période de l'Antiquité, du Moyen-âge en France et de l'ère préindustrielle et industrielle, tant en France qu'au Québec. On y constate à quel point la façon dont les hommes exercent leur paternité est un construit social et historique, variant d'une époque à une autre, d'une civilisation à une autre. Cette perspective permet également de prendre la mesure de la diversité de paternités possibles à la suite de l'éclatement de normes, notamment judéochrétiennes. Cet assouplissement des attentes sociales face à la façon d'être père, tout comme celle d'être mère, fait aussi place à de nouveaux phénomènes sociaux qui ne sont pas vécus sans heurts. Notamment, les auteurs insèrent la paternité dans des conjonctures telles que le divorce, la monoparentalité, les défis engendrés par la garde partagée, particulièrement en contexte de pauvreté, phénomènes occasionnant du désengagement de la part des pères et ayant des effets négatifs sur les enfants. Les situations de pères auxquelles font face les travailleurs sociaux œuvrant dans le champ de la famille et de l'enfance sont illustrées de nombreux exemples. En somme, cet article permet de situer la paternité contemporaine dans un contexte historique large qui en constitue 
la toile de fond et d'en saisir les effets sur les familles. Cette lecture interroge le travail social dans la recherche de pratiques pouvant répondre à ces nouveaux besoins.

Parmi les réalités peu étudiées dans le champ de la paternité, on retrouve celle qui survient chez les jeunes hommes et les adolescents. Nous lui consacrons un article que nous signons nous-même et qui porte sur la façon dont des jeunes hommes qui vont devenir pères vivent la période prénatale. Nous avons réalisé une recherche auprès de trente jeunes futurs pères dont la compagne était âgée de moins de vingt ans à la date prévue de l'accouchement. Il en ressort notamment qu'une fois la poursuite de la grossesse confirmée, ils sont généralement déterminés à s'acquitter des responsabilités liées au rôle de père. Ils se préparent à assumer ce rôle en dépit de conditions défavorables, telles que leur jeune âge, leur faible scolarité, leur situation de pauvreté. Contrairement aux croyances à leur sujet, cette étude indique que beaucoup de futurs jeunes pères sont présents dans la vie de la jeune mère durant la grossesse. Également, pour la plupart d'entre eux, bien que souvent peu planifiée, la grossesse devient un projet de vie, même s'il est parfois difficile à concrétiser. Ces résultats incitent à prendre un recul face à un courant de recherche s'inscrivant dans un paradigme déficitaire, c'est-à-dire qui met en lumière les facteurs de risque présents chez les jeunes pères, sans tenir compte de leurs forces et du sens qu'ils donnent à leur situation. Ces constats sont pertinents pour l'intervention sociale qui doit non seulement tenir compte de leurs difficultés, mais aussi de leurs aspirations.

Dans leur article, Josianne Chartrand et Marie Drolet explorent sous un angle à la fois théorique et pratique une dimension méconnue de la paternité : la notion de genre dans l'intervention auprès des enfants manifestant des comportements sexuels problématiques et auprès de leurs parents. Leur contribution prend la forme d'une recension des écrits portant sur les enfants présentant des comportements sexuels inappropriés à l'endroit d'autres enfants. Leur recherche documentaire a amené les auteures à conclure que la notion de genre est occultée dans la conceptualisation de cette problématique et, par le fait même, 
des programmes d'intervention destinés à cette clientèle. Pourtant, les auteurs de gestes sexuels inadaptés sont des garçons. On se demande quelle est la place du père dans la compréhension de cette problématique. On connaît le rôle central que le père occupe en tant que modèle comportemental pour son fils, tant sur le plan de son impact sur l'apparition des conduites inappropriées qu'en ce qui a trait au potentiel qu'il représente pour l'apprentissage de comportements plus sains. Dans le but de bonifier l'intervention en la matière, l'article soulève une réflexion sur des questions délicates, dont les besoins spécifiques des garçons et les façons d'intervenir auprès des pères pour favoriser un changement de comportement. En soi, il s'agit d'une question importante puisque les services sociaux qui leur sont destinés font défaut. Chercher des façons pour les pères de participer à la solution constitue une stratégie peu envisagée, pourtant cruciale. De plus, les auteures soulignent la pertinence de développer une approche familiale pour intervenir dans ces situations. Cet angle est également très original, car il soulève la possibilité de dépasser les interventions sociojudiciaires qui, bien que nécessaires, ne peuvent à elles seules accompagner les familles dans un changement plus profond.

Toujours dans l'optique d'étudier des aspects méconnus de la paternité, Christine Gervais et Francine de Montigny proposent un article qui traite de deux dimensions aussi importantes que peu abordées, soit celle de la transition à la paternité dans un contexte d'immigration récente et celle du point de vue d'hommes sur l'allaitement. Cette étude porte sur les croyances des pères originaires du Maghreb envers l'allaitement maternel ainsi que sur les facteurs facilitant l'expérience de l'allaitement. Dans cette optique, les auteures indiquent l'émergence d'une perspective de recherche qui inclut les pères dans la recherche de mesures et de pratiques dans le champ de la santé publique et de la famille et de la petite enfance. Il s'agit en soi d'axes de recherche aussi importants qu'inexplorés. Effectivement, on parle très peu des pères immigrants malgré l'apport démographique majeur des couples et des familles qui viennent s'installer au pays. Il en est de même de la contribution du père au bien-être psychosocial et physique de la mère et du nourrisson alors que les enfants sont en 
très bas âge. Pourtant, comme société, on cherche à encourager les pères à être les plus présents possible durant cette période. Ainsi, en rapportant les propos de pères portant sur l'intérêt que des pères immigrants accordent, entre autres, à l'allaitement maternel, les auteures documentent un aspect de cette question et formulent des recommandations pour les pratiques auprès des familles immigrantes, tant pour la recherche que pour la formation de futurs intervenants.

La section que Reflets consacre aux pratiques pousse jusqu'au contexte militaire l'exploration des paternités méconnues. Karine Régimbald et nous-même signons une synthèse des écrits portant sur différents facteurs qui influencent l'exercice de la paternité chez les militaires canadiens afin d'en comprendre les effets sur leur famille. Certains de ces facteurs entravent l'exercice de la paternité chez les soldats canadiens. D'autres le facilitent. Il en ressort un portrait complexe qui, bien qu'il soit difficile de le généraliser à tous les militaires, démontre que la vie de père et la vie de famille sont influencées par plusieurs facteurs propres aux pères soldats. La façon de jouer leur rôle est influencée par leur mode de vie et par la culture du milieu, lesquels imposent des politiques et des services offerts spécifiquement aux familles militaires. Présenter cette réalité implique de faire état de l'impact du mode de vie des militaires sur les mères et les enfants. Une perspective familiale est donc privilégiée pour présenter la façon dont la paternité est intimement liée aux conditions auxquelles sont confrontées les familles de militaires. Enfin, l'article se veut une occasion de proposer quelques moyens de mieux soutenir les pères militaires au sein des Forces canadiennes en portant une attention particulière au rôle des travailleurs sociaux à leur égard.

Ce numéro se termine sur un article hors thème dans lequel Caroline Veilleux et Marc Molgat rapportent les résultats d'une recherche portant sur la réalité de jeunes adultes ayant reçu un diagnostic de maladie mentale à partir de l'étude de leur discours. Il vise dans un premier temps à déterminer si ces jeunes adultes se perçoivent comme des adultes et à identifier les critères associés à cette perception d'être adultes. On y aborde aussi l'impact de la maladie mentale sur la perception de soi en tant que jeune adulte, 
la représentation et le positionnement de ces jeunes adultes par rapport aux services de santé mentale. L'analyse approfondie du discours révèle que pour expliquer leur parcours de vie ces jeunes adultes ne s'appuient pas seulement sur les marqueurs de transition formels, tels que l'entrée dans le marché du travail et la parentalité. Ils s'en remettent davantage à leurs expériences personnelles de vie : ces jeunes établissent plutôt de nouveaux points de repère pour donner un sens à leur réalité. En conclusion à leur article, les auteurs proposent quelques pistes d'intervention. 